\title{
Cytoreductive nephrectomy: a treatment of the past
}

\author{
Peter Venner, MD, FRCP
}

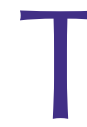
he premise that a cytoreductive nephrectomy $(\mathrm{CN})$ in the presence of metastatic renal cell carcinoma (mRCC) is of any value other than for palliation needs to be carefully reconsidered. Now that there is therapy that is much more efficacious than cytokine therapy, we need to be much more circumspect when considering the role of a $\mathrm{CN}$ in a patient with metastatic disease. Delaying or even failing to initiate effective therapy as quickly as is medically indicated, while intervening with what is now unproven intervention, is foolhardy indeed.

What is the case to support the role of $\mathrm{CN}$ ? There are 2 randomized studies that investigated the role of $\mathrm{CN}$ preceding immunotherapy that were done almost simultaneously. 1,2 They had the same study design, treatment arms, subject eligibility criteria, sample size calculations for the primary endpoint of survival; there were also published within months of each other. With deference to the authors and participants of the pivotal studies that were done in the late $20^{\text {th }}$ century it must be conceded that studies such as these, which randomize subjects to a major surgery, are very difficult to do and they are to be commended for taking on this very difficult endeavour. However, in critical review of these studies I believe that the conclusions are not supported by the data.

There are several criticisms that can be raised. There was an imbalance of patients with good performance status (PS) between the 2 arms; a number of patients were not able to receive the planned treatment (although intent to treat analyses were performed) and one study was so severely underpowered that, not only should it not have been accepted for publication, no credence should be placed on the conclusion drawn by the authors.

The larger of these studies was undertaken by the Southwest Oncology Group (SWOG) and reported in 2001. 1 The study successfully accrued the planned sample size of 244 subjects, although it did take them 7 years even with 80 centres participating (an average of 0.4 patients per centre, per year). Sample size determinations are very much dependent on planned accrual rates. Although there is no mention of collaboration with the European Organization for Research and Treatment of Cancer (EORTC) or whether or not there was to be a combined analysis of the subjects enrolled into both studies. The EROTC paper mentions collaboration for accrual and publication with SWOG.

There is no question that the SWOG study resulted in a statistically and clinically significant difference in survival in favour of those that had a nephrectomy prior to systemic therapy with interferon. However I am concerned that there were either flaws in the stratification process or by chance there was an imbalance of patients with a better SWOG PS in the CN arm that may have unwittingly, but meaningfully, biased the results in favour of the intervention arm.

It is stated in both publications that patients were stratified according to PS (PS 0 vs. PS 1), however there is a disproportionate number of PS 0 patients in the nephrectomy and interferon (IFN) arm, and a similar disproportionate number of PS 1 in IFN-alone arm. In the CN arm, 53\% of the patients had a PS 0 , whereas only $41 \%$ of the patients in the IFN-alone arm had a PS 0 . In relative terms, this is $30 \%$ more patients with better PS in the $\mathrm{CN}$ arm. It therefore follows that there were more patients $(12 \%)$ with a PS 1 in the IFN-alone arm, which in relative terms means that there were $26 \%$ more patients in this arm with a worse PS. What is the significance of this small difference in PS? Based on knowledge that has been around since the 1980s, it is well known that the survival of patients with mRCC with PS 0 is significantly worse than those with a PS 1. In 1988 Elson and colleagues reported that patients with a PS 0 had a median survival of 10.2 months compared to 6.7 months in patients with a PS 1.3 These survival figures are very similar to the overall results reported in the SWOG study (11.1 vs. 8.1 months). In 1995, Mani reported an even more dramatic difference in median survival for patients with a PS 0 compared to PS 1 (15.2 and 6 months, respectively). ${ }^{4}$ Flanigan reports that the survival difference was not significant when the analyses were based on the PS stratification. ${ }^{1}$ There was a similar imbalance in favour of the $\mathrm{CN}$ arm in the EORTC study though it was not as remarkable (possibly related to the small sample size). ${ }^{2}$ In a subsequent report combining the SWOG and the EORTC studies, this imbalance in favour of the $\mathrm{CN}$ arm persists and patients with a PS 0 had a significantly longer survival. ${ }^{5}$

With regard to the inadequate sample size in the EROTC study, there does not seem to be an adequate explanation 
for this. It is clearly stated in the paper that they planned to recruit 80 patients to the SWOG study and that the results of this study should not be interpreted in isolation from the SWOG study. So why did they publish this study separately and why was it accepted for publication? It is clearly stated that they required 244 patients to demonstrate a clinically meaningful and significant improvement in survival with $\mathrm{CN}$ combined with IFN therapy; this was to be done in 3 years. In the end, they recruited only 80 patients in 3 years. There is also concern with the sample size in the SWOG study, despite it reaching the accrual target. Patient recruitment was also supposed to be completed in 3 years, but it took over 7 years. In addition, there were 80 centres in the study, averaging 3 patients per centre and 0.4 patients per year per centre. When accrual is prolonged, the sample size has to increase and, when recalculated for 4 delays in reaching accrual, the sample size should have increased to more than 4 subjects (one-sided) or more than 500 (twosided). In this same study, $17 \%$ of the patients did not have a nephrectomy or have interferon, which does appear to have been accounted for in the sample size calculation.

Finally, the most important reason not to perform a nonpalliative $\mathrm{CN}$ is that the playing field for the systemic therapy of mRCC has markedly changed from the cytokine era of the last century. Although far from curative, the therapies we now have meaningfully prolong survival and a recognizable proportion of patients have long-term survival. Although in the clinical trials of the targeted therapies about $90 \%$ of the patients have had a nephrectomy, this should not imply that all patients undergoing targeted therapy should have a nephrectomy. ${ }^{6,7}$ Our own limited data support the concept that the time from diagnosis to treatment is a more important prognostic factor than whether or not a nephrectomy has been performed. ${ }^{8,9}$ It would be a tragedy if a patient was advised to have a nephrectomy and, due to disease progression or complications of surgery, the patient was denied the opportunity to go onto targeted therapy.

In summary, $\mathrm{CN}$ is a treatment of the past. Cytoreductive nephrectomy offered a glimmer of hope in a time when we had limited therapies for mRCC. Now, as with all other cancers who present with metastatic disease, cytoreductive surgery should not be offered to patients as there is more effective therapy available to them. They should not be denied this life-prolonging therapy. A chance to cut is not a chance to cure in mRCC.

From the Department of Medical Oncology, Cross Cancer Institute, Division of Medical Oncology, Department of Oncology, University of Alberta, Edmonton, AB

Competing interests: None declared.

This paper has been peer-reviewed.

\section{References}

1. Flanigan RC, Salmon SE, Blumenstein BA, et al. Nephrectomy followed by interferon alfa-2b compared with interferon alfa-2b alone for metastatic renal-cell cancer. N Engl J Med 2001;345:1655-9.

2. Mickisch GH, Garin A, van Poppel H, et al..; European Organisation for Research and Treatment of Cancer (EORTC) Genitourinary Group. Radical nephrectomy plus interferon-alfa-based immunotherapy compared with interferon alfa alone in metastatic renal-cell carcinoma: a randomised trial. Lancet 2001;358:966-70.

3. Elson PJ, Witte RS, Trump DL. Prognostic factors for survival in patients with recurrent or metastatic renal cell carcinoma. Cancer Res 1988;48(24Pt1):7310-3.

4. Mani S, Todd MB, Katz K, et al. Prognostic factors for survival in patients with metastatic renal cancer treated with biological response modifiers. J Urol 1995; 154:35-40.

5. Flanigan RC, Mickisch G, Sylvester R, et al. Cytoreductive nephrectomy in patients with metastatic renal cancer: a combined analysis. J Urol 2004;171:1071-6.

6. Mickisch GH, Garin A, van Poppel H, et al.; European Organisation for Research and Treatment of Cancer (EORTC) Genitourinary Group. Radical nephrectomy plus interferon-alfa-based immunotherapy compared with interferon alfa alone in metastatic renal-cell carcinoma: a randomised trial. Lancet 2001;358:966-70.

7. Escudier B, Eisen T, Stadler WM, et al.; TARGET Study Group. Sorafenib in advanced clear-cell renal-cell carcinoma. N Engl J Med 2007;356:125-34. Erratum in: N Engl J Med 2007;357:203.

8. Motzer RJ, Bacik J, Murphy BA, et al. Interferon-alfa as a comparative treatment for clinical trials of new therapies against advanced renal cell carcinoma. J Clin Oncol 2002;20:289-96.

9. Warren M, Venner PM, North S, et al. A population-based study examining the effect of tyrosine kinase inhibitors on survival in metastatic renal cell carcinoma in Alberta and the role of nephrectomy prior to treatment. Can Urol Assoc J 2009;4:281-9.

Correspondence: Dr. Peter Venner, Division Director, 11560 University of Alberta, Edmonton, AB T6G 1Z2; peterven@cancerboard.ab.ca 